\title{
Adherence level of Health care Providers to first visit antenatal care guideline and its effect on perinatal outcome in Tigray public Health institutions, 2017/18: Cohort study
}

\section{CURRENT STATUS: POSTED}

2 Research Square

Abera Haftu Melkamu

Mekelle University

aberahaftu@gmail.comCorresponding Author

Tesfay Adhena Hailu

"axum university

Brhane G/her G/micheal

Mekelle University

Hadgay Hagos Adhanu

Mekelle University

Mhiret-AB Mehari Reda

Mekelle University

Assefa Hagos Kebede

Mekelle University

Tsige Araya Hailu

Mekelle University

Hailemaryam Berhe Kahsa

Mekelle University

Haftu Berhe Gebru

Mekelle University

Alemayehu Bayray Kahsay

Mekelle University

DOI: 
$10.21203 / \mathrm{rs} .2 .22327 / \mathrm{v} 1$

\section{SUBJECT AREAS}

Sexual \& Reproductive Medicine

KEYWORDS

antenatal, adherence, perinatal outcome, providers 
Abstract

Background: Antenatal care is one of the medical services delivered to pregnant women for the safety of the mother and the neonate. Guidelines are developed to ensure quality, uniformity and consistency of care for clients. One of the guidelines is focused antenatal care. Despite this fact, there is a paucity of information regarding the adherence of health professionals in the implementation of the guidelines and its effect on the perinatal outcomes.

Objective: To assess adherence level of health care providers to first visit antenatal care guidelines and its effect on perinatal outcomes among mothers in Tigray Public health institutions in 2017/18. Methods: Cohort study design was employed with sample size of 1545. Among the participants 501(496 with complete follow up) were recruited to exposed groups and 1044 for non-exposed groups. The ratio of exposed to non-exposed group was 1:2. In the selection of study sites simple random sampling method was used but to select study participants consecutive sampling technique was employed. Women with full adherence to the first antenatal guideline were considered as exposed group, whereas those with incomplete adherence were considered as non- exposed group. It was assumed that the risk of complication among the non-exposed group was twice as that of the exposed group. Data was entered to Epi data version 3.51 and exported to SPSS version 20 for further analysis. Binary logistic regression was employed to determine the associated factors and multivariable analysis was done to control the confounding factors and significance was declared at $\mathrm{Cl}$ of $95 \%$ and $p$-value of $<0.05$. Relative risk was used to express the association and effect of adherence on the perinatal outcome.

Result: Overall, the level of complete adherence to antenatal care guideline was $32.2 \%$.The risks of antenatal, intranatal and neonatal complication was $21 \%, 41.3 \%$ and $26.8 \%$ respectively. The risk of maternal and neonatal complication is higher in non-exposed groups, pregnancy induced hypertension and postpartum hemorrhage risks were 1.6\%:6.2\% and 2.6\%:4.3\% among exposed and non-exposed groups and a neonatal complication of low birth weight and preterm labor was 5.8\%:8.2\% and 6.7\%:8\% among exposed and non-exposed groups respectively. Effect of complete adherence was found to be significant in reducing maternal and neonatal complications. Neonatal 
complications, anemia, postpartum hemorrhage and early neonatal death was reduced by $40 \%$ among the women with complete adherence, maternal sepsis and neonatal sepsis were reduced by $60 \%$ among the exposed groups and pregnancy induced hypertension was reduced by about $70 \%$ in women with complete adherence.

Conclusion and recommendations: Overall providers' complete adherence to antenatal care guideline at first visit influences both maternal and neonatal outcomes. On average the risks of developing antenatal, intranatal and post natal complications were reduced by almost $50 \%$ among the exposed groups. The Federal Ministry of Health and teaching institutions should capacitate their staff to strictly adhere to the implementation of the guidelines

\section{Full Text}

Due to technical limitations, full-text HTML conversion of this manuscript could not be completed. However, the manuscript can be downloaded and accessed as a PDF.

Figures

\section{postnatal follow up}

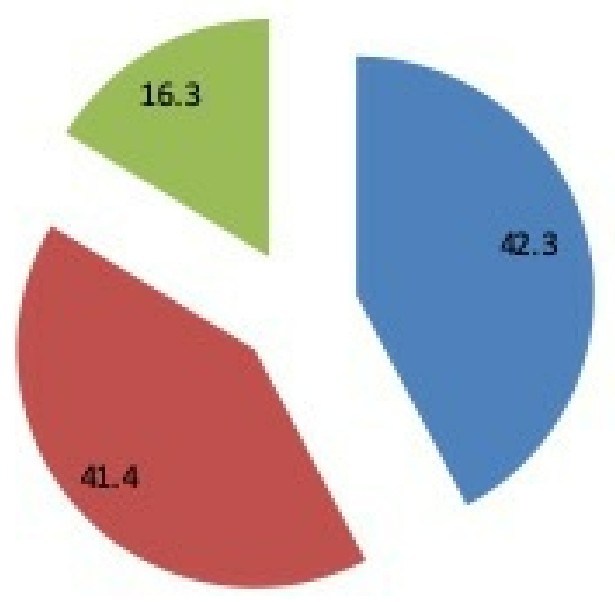

Figure 1

Postnatal follow up women's who gave birth in Tigray public health institutions 


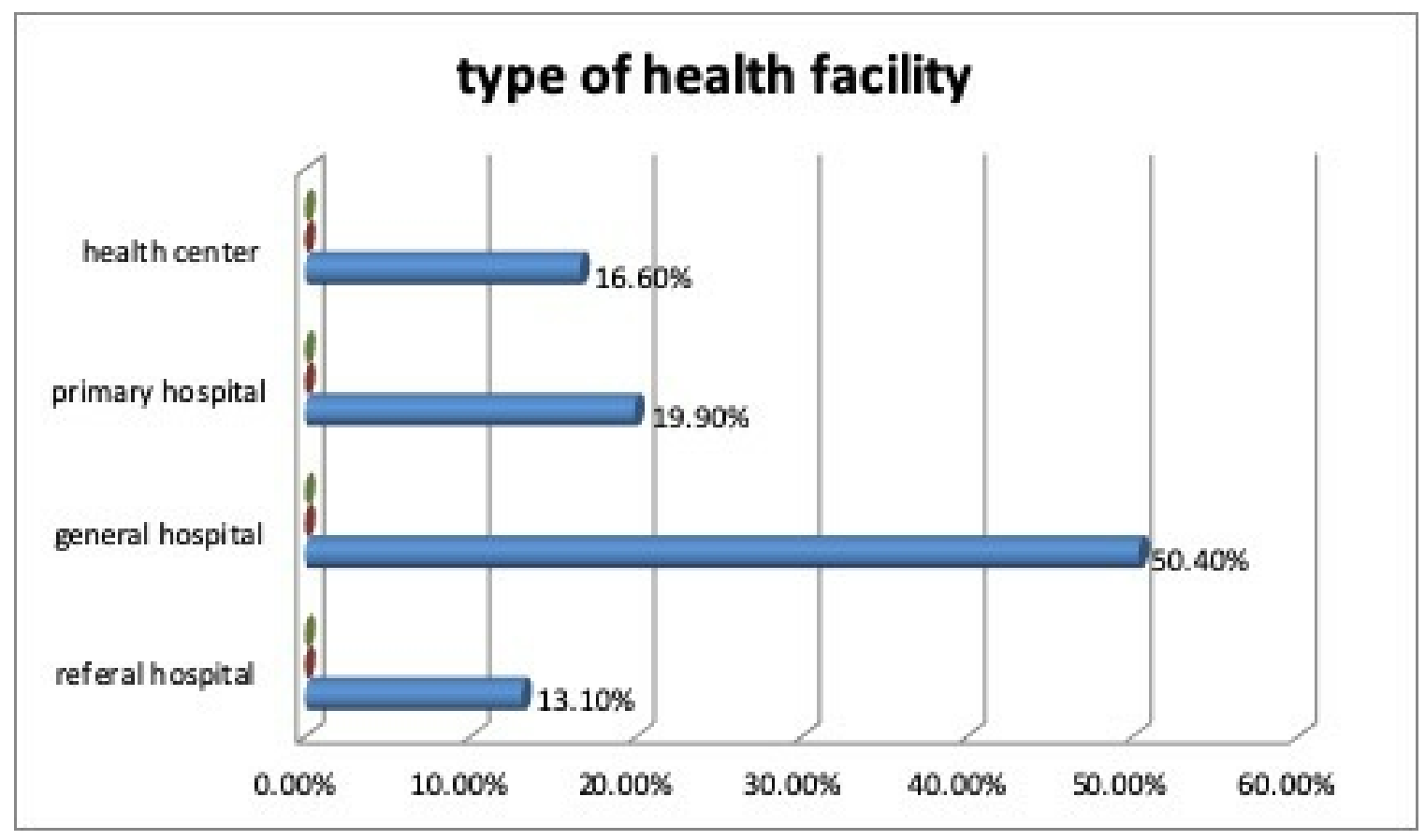

Figure 2

Type of health facility where women's gave birth in Tigray public health institutions 